\title{
Phenotypic diversity in cold-tolerant peanut (Arachis hypogaea L.) germplasm
}

\author{
H. D. Upadhyaya $\cdot$ L. J. Reddy $\cdot$ S. L. Dwivedi • \\ C. L. L. Gowda $\cdot$ S. Singh
}

Received: 19 August 2007/Accepted: 7 August 2008/Published online: 2 September 2008

(C) The Author(s) 2008. This article is published with open access at Springerlink.com

\begin{abstract}
Tolerance to low temperature is an important prerequisite for optimal performance of peanut (Arachis hypogaea L.) in a number of temperate peanut-growing environments. One hundred fifty-eight peanut accessions belonging to five botanical types, known to be tolerant to low temperature $\left(12^{\circ} \mathrm{C}\right)$ at germination, were evaluated for phenotypic diversity for 15 morphological traits in the 2001 rainy season and for 15 agronomic and two seed quality traits in the 2001 rainy and 2001/2002 post-rainy seasons. Analysis of data, using the residual maximum-likelihood approach indicated that variance components due to genotypes were significant for all traits in the rainy and for all but two traits in the post-rainy season. Clustering based on scores of nine principle components delineated four clusters. The cold-tolerant genotypes and the standard control cultivars in the four clusters differed in mean, variance, and range both during rainy and post-rainy seasons for a range of agronomic traits, indicating the diversity among the clusters. The cold-tolerant accessions were superior to control cultivars for several agronomic traits compared with their respective controls in both the rainy and post-rainy seasons,
\end{abstract}

H. D. Upadhyaya (ه) · L. J. Reddy ·

S. L. Dwivedi - C. L. L. Gowda · S. Singh International Crops Research Institute for the Semi-Arid Tropics (ICRISAT), Patancheru 502324, India e-mail: h.upadhyaya@cgiar.org so their use in breeding should result in genetically diverse cold-tolerant high-yielding peanut cultivars.

Keywords Groundnut - Cold tolerance ·

Genetic diversity · Principal component analysis

\section{Introduction}

Peanut (Arachis hypogaea L.), also known as groundnut, is an important tropical legume grown for both oil production and human food, as it provides a good source of energy, protein, minerals, and vitamins. Peanut production environments are characterized by a warm, frost-free period of at least 90 days (Bunting et al. 1985), with mean temperatures between $24^{\circ} \mathrm{C}$ and $33^{\circ} \mathrm{C}$, which is the optimum range for growth and dry-matter production (Ketring 1984). The peanut plant shows maximum growth at $28^{\circ} \mathrm{C}$ but experiences severe metabolic perturbations below $12^{\circ} \mathrm{C}$ (Bell et al. 1994a). Low temperature results in slow growth of both hypocotyl/radicle and epicotyl (Ketring et al. 1982). Night temperature determines both leaflet $\mathrm{CO}_{2}$ exchange rate, regardless of day temperature, and the efficiency of use of intercepted photosynthetically active radiation (Sinclair et al. 1993; Bell et al. 1994b). Low soil temperature delays pod initiation, and reduces number of mature pods/seeds, and seed weight (Golombok and Johanson 1997). 
The importance of cold tolerance in peanut is well recognized for specific production environments in North America (Benedict and Ketring 1972; Singleton and Pattee 1989; Bell et al. 1994a), Australia (Bell et al. 1991), India (Bhagat et al. 1992), and China (Fu et al. 1988). A few cold-tolerant earlymaturing cultivars with ability to germinate in cooler soils have been released in Canada (Agriculture Canada 1984,1989$)$. The low temperatures $\left(<18^{\circ} \mathrm{C}\right)$ at sowing in the winter peanut crop in India result in slow seedling emergence and poor plant stand. Delay in seedling emergence extends crop duration beyond 120 days, exposing the crop to high temperatures at reproductive phase and pod damage due to early onset of monsoon rains (Bhagat et al. 1988). Poor germination due to low temperatures in spring-sown peanut crop has also been reported in China, Nepal, and Vietnam (Fu et al. 1988; Koirala 1996; Dan and Hong 1996). Identification and incorporation of cold tolerance are therefore important peanut breeding objectives in these countries.

Upadhyaya et al. (2001) screened 1,704 peanut core collection accessions (Upadhyaya et al. 2003) and four control cultivars (Gangapuri, M13, ICGS 44, and ICGS 76), using rolled paper towel testing (Ellis et al. 1985) for ability to germinate in an incubator set at $12^{\circ} \mathrm{C}$ day-night temperature. Seeds of hypogaea and hirsuta were treated with ethrel (2-chloroethylphosphonic acid) to break seed dormancy prior to cold-tolerant test. A sufficient quantity of distilled water was added to the tray so as to keep wet the 3$4 \mathrm{~cm}$ of paper rolls. The number of germinating seeds was recorded at 10 (fastigiata, vulgaris, aequatoriana, and peruviana types) and 15 (hypogaea and hirsuta types) days after incubation. The hypogaea types that showed less than $70 \%$ germination were retested, with the seed stored for at least 6 months, to avoid discrepancies that could arise due to seed dormancy. This experiment was repeated with the same number of entries during the 2000/2001 postrainy season. In both seasons, accessions that showed $80 \%$ or higher germination for one seed source but not less than $70 \%$ from either seed source were considered tolerant to low temperature at germination. Based on two seasons' evaluation, 158 peanut core collection accessions were identified as tolerant to low temperature $\left(12^{\circ} \mathrm{C}\right)$, on the basis of $80 \%$ or higher germination for the best seed source and not less than $70 \%$ from the second source. Mean percentage seed germination in cold-tolerant entries ranged from $76 \%$ to $96 \%$, compared with $36 \%$ to $55 \%$ in controls (M13, ICGS 44, and ICGS 76); the fourth control, Gangapuri, had $87 \%$ germination at $12^{\circ} \mathrm{C}$ (Table 1).

The present study was done to characterize phenotypic diversity for morphological and agronomic traits in the 158 cold-tolerant germplasm to identify genetically diverse accessions for use in peanut breeding to improve cold tolerance at germination.

\section{Materials and methods}

One hundred fifty-eight cold-tolerant peanut accessions, representing five botanical types (4 aequatoriana, 103 fastigiata, 11 peruviana, 5 vulgaris, and 35 hypogaea) and four released Indian control cultivars (Gangapuri, M13, ICGS 44, and ICGS 76) were evaluated for 15 morphological traits in field plantings in the 2001 rainy season and for 15 agronomic and two seed quality traits in the 2001/ 2002 post-rainy season at ICRISAT, Patancheru, India. Gangapuri (ICG 2738) belongs to subsp. fastigiata var. fastigiata (Valencia type) and matures in about 100 days. ICGS 44 (ICG 13941) belongs to subsp. fastigiata var. vulgaris (Spanish type), matures in about 120 days, and is adapted to the irrigated post-rainy season. Both M13 (ICG 156) and ICGS 76 (ICG 13942) belong to subsp. hypogaea var. hypogaea (Virginia type), mature in 120-135 days, and are adapted to rainy season conditions.

The experiment was conducted in an alpha design (Paterson and Williams 1976) with two replications in the rainy season and three replications in postrainy season. Each accession was sown in a one row plot of $4 \mathrm{~m}$ length, with $60 \mathrm{~cm}$ between rows and $10 \mathrm{~cm}$ between plants in both the seasons. Morphological descriptors used included growth habit, branching pattern, stem color, stem hair, leaflet color, leaflet shape, leaflet hair, flower color, streak color on flower, peg color, seeds per pod, pod beak, pod constriction, pod reticulation, and primary seed color (IBPGR and ICRISAT 1992). Ten mature pods were randomly selected to record data on pod beak, constriction, and reticulation. Days to emergence, days to $50 \%$ flowering, pod yield per plot, pod length and width, seed length and width, and shelling 
Table 1 Identity, country of origin, and germination (\%) at $12^{\circ} \mathrm{C}$ in $1999 / 2000$ and $2000 / 2001$ post-rainy seasons harvested seeds in 158 cold-tolerant germplasm and control cultivars in peanut

\begin{tabular}{|c|c|c|c|c|}
\hline \multirow[t]{2}{*}{ Identity } & \multirow[t]{2}{*}{ Origin } & \multicolumn{3}{|c|}{ Germination $(\%)$} \\
\hline & & $\begin{array}{l}1999 / 2000 \\
\text { post-rainy }\end{array}$ & $\begin{array}{l}2000 / 2001 \\
\text { post-rainy }\end{array}$ & Mea \\
\hline \multicolumn{5}{|c|}{ var. aequatoriana } \\
\hline ICG 7898 & Ecuador & 86 & 78 & 82 \\
\hline ICG 12553 & Ecuador & 90 & 86 & 88 \\
\hline ICG 12625 & Ecuador & 88 & 78 & 83 \\
\hline $\begin{array}{l}\text { ICG } 12719 \\
\text { var. fastigiat }\end{array}$ & Ecuador & 84 & 76 & 80 \\
\hline ICG 115 & India & 86 & 100 & 93 \\
\hline ICG 282 & USA & 98 & 98 & 98 \\
\hline ICG 318 & Brazil & 82 & 88 & 85 \\
\hline ICG 376 & Argentina & 94 & 84 & 89 \\
\hline ICG 389 & South Africa & 92 & 88 & 90 \\
\hline ICG 397 & USA & 94 & 82 & 88 \\
\hline ICG 398 & USA & 92 & 80 & 86 \\
\hline ICG 445 & Tanzania & 82 & 96 & 89 \\
\hline ICG 457 & USA & 92 & 86 & 89 \\
\hline ICG 1158 & India & 92 & 94 & 93 \\
\hline ICG 1256 & Uganda & 86 & 100 & 93 \\
\hline ICG 1274 & Indonesia & 84 & 92 & 88 \\
\hline ICG 1298 & Unknown & 84 & 100 & 92 \\
\hline ICG 1384 & Tanzania & 86 & 98 & 92 \\
\hline ICG 1399 & Malawi & 92 & 70 & 81 \\
\hline ICG 1683 & South Africa & 94 & 72 & 83 \\
\hline ICG 1796 & Senegal & 94 & 98 & 96 \\
\hline ICG 1824 & Zaire & 90 & 84 & 87 \\
\hline ICG 1899 & Uganda & 92 & 98 & 95 \\
\hline ICG 1908 & India & 90 & 76 & 83 \\
\hline ICG 2039 & Unknown & 82 & 70 & 76 \\
\hline ICG 2057 & China & 88 & 94 & 91 \\
\hline ICG 2145 & Sudan & 90 & 76 & 83 \\
\hline ICG 2158 & Uganda & 98 & 75 & 87 \\
\hline ICG 2159 & Sierra Leone & 88 & 88 & 88 \\
\hline ICG 3125 & Sudan & 90 & 80 & 85 \\
\hline ICG 3219 & Tanzania & 88 & 94 & 91 \\
\hline ICG 3477 & India & 86 & 100 & 93 \\
\hline ICG 3510 & Argentina & 90 & 72 & 81 \\
\hline ICG 3726 & India & 92 & 96 & 94 \\
\hline ICG 3779 & Tanzania & 86 & 72 & 79 \\
\hline ICG 4087 & USA & 88 & 90 & 89 \\
\hline ICG 4670 & Sudan & 96 & 92 & 94 \\
\hline ICG 4788 & Benin & 82 & 92 & 87 \\
\hline
\end{tabular}

Table 1 continued

\begin{tabular}{|c|c|c|c|c|}
\hline \multirow[t]{2}{*}{ Identity } & \multirow[t]{2}{*}{ Origin } & \multicolumn{3}{|c|}{ Germination $(\%)$} \\
\hline & & $\begin{array}{l}\text { 1999/2000 } \\
\text { post-rainy }\end{array}$ & $\begin{array}{l}2000 / 2001 \\
\text { post-rainy }\end{array}$ & Mean \\
\hline ICG 4890 & Argentina & 94 & 98 & 96 \\
\hline ICG 4992 & USA & 94 & 92 & 93 \\
\hline ICG 5094 & Brazil & 84 & 86 & 85 \\
\hline ICG 5475 & Kenya & 94 & 88 & 91 \\
\hline ICG 5609 & Sri Lanka & 90 & 92 & 91 \\
\hline ICG 5964 & Zimbabwe & 86 & 92 & 89 \\
\hline ICG 6022 & Sudan & 96 & 92 & 94 \\
\hline ICG 6148 & USA & 98 & 94 & 96 \\
\hline ICG 6203 & Zimbabwe & 86 & 90 & 88 \\
\hline ICG 6220 & Brazil & 88 & 94 & 91 \\
\hline ICG 6221 & Brazil & 86 & 84 & 85 \\
\hline ICG 6340 & Honduras & 90 & 90 & 90 \\
\hline ICG 6421 & Malawi & 86 & 88 & 87 \\
\hline ICG 6565 & Unknown & 84 & 96 & 90 \\
\hline ICG 6570 & Unknown & 96 & 100 & 98 \\
\hline ICG 6706 & Brazil & 90 & 84 & 87 \\
\hline ICG 6725 & Argentina & 90 & 94 & 92 \\
\hline ICG 6878 & Argentina & 98 & 98 & 98 \\
\hline ICG 6888 & Brazil & 94 & 100 & 97 \\
\hline ICG 7005 & Brazil & 88 & 100 & 94 \\
\hline ICG 7013 & India & 86 & 92 & 89 \\
\hline ICG 7285 & Zimbabwe & 98 & 94 & 96 \\
\hline ICG 7352 & Peru & 94 & 96 & 95 \\
\hline ICG 7355 & Paraguay & 90 & 96 & 93 \\
\hline ICG 7777 & Unknown & 84 & 98 & 91 \\
\hline ICG 7812 & Brazil & 82 & 86 & 84 \\
\hline ICG 7884 & Israel & 96 & 92 & 94 \\
\hline ICG 7905 & Zimbabwe & 94 & 88 & 91 \\
\hline ICG 7929 & Paraguay & 94 & 100 & 97 \\
\hline ICG 7978 & Russia \& CIS & 92 & 100 & 96 \\
\hline ICG 8360 & Thailand & 84 & 98 & 91 \\
\hline ICG 8485 & Zimbabwe & 84 & 74 & 79 \\
\hline ICG 8514 & South Africa & 84 & 84 & 84 \\
\hline ICG 8517 & Bolivia & 86 & 94 & 90 \\
\hline ICG 8570 & Argentina & 92 & 88 & 90 \\
\hline ICG 9141 & Zaire & 88 & 96 & 92 \\
\hline ICG 9144 & Syria & 84 & 98 & 91 \\
\hline ICG 9929 & Zimbabwe & 88 & 96 & 92 \\
\hline ICG 10075 & Peru & 90 & 70 & 80 \\
\hline ICG 10092 & Zimbabwe & 88 & 76 & 82 \\
\hline ICG 10371 & Nigeria & 84 & 92 & 88 \\
\hline ICG 10402 & USA & 84 & 78 & 81 \\
\hline
\end{tabular}


Table 1 continued

\begin{tabular}{|c|c|c|c|c|}
\hline \multirow[t]{2}{*}{ Identity } & \multirow[t]{2}{*}{ Origin } & \multicolumn{3}{|c|}{ Germination $(\%)$} \\
\hline & & $\begin{array}{l}\text { 1999/2000 } \\
\text { post-rainy }\end{array}$ & $\begin{array}{l}\text { 2000/2001 } \\
\text { post-rainy }\end{array}$ & Mean \\
\hline ICG 10481 & Venezuela & 82 & 82 & 82 \\
\hline ICG 10495 & Paraguay & 90 & 72 & 81 \\
\hline ICG 10519 & Australia & 86 & 80 & 83 \\
\hline ICG 10549 & Argentina & 96 & 90 & 93 \\
\hline ICG 10554 & Argentina & 90 & 82 & 86 \\
\hline ICG 10566 & Congo & 92 & 94 & 93 \\
\hline ICG 10595 & Brazil & 86 & 80 & 83 \\
\hline ICG 10616 & Argentina & 98 & 92 & 95 \\
\hline ICG 10788 & Tanzania & 92 & 96 & 94 \\
\hline ICG 10900 & Peru & 84 & 98 & 91 \\
\hline ICG 11130 & Brazil & 94 & 74 & 84 \\
\hline ICG 11203 & India & 86 & 90 & 88 \\
\hline ICG 11605 & Bolivia & 86 & 70 & 78 \\
\hline ICG 12498 & Brazil & 92 & 74 & 83 \\
\hline ICG 12564 & Uruguay & 92 & 70 & 81 \\
\hline ICG 12665 & Peru & 88 & 88 & 88 \\
\hline ICG 12743 & Bolivia & 92 & 96 & 94 \\
\hline ICG 12963 & Zimbabwe & 86 & 90 & 88 \\
\hline ICG 13049 & India & 90 & 86 & 88 \\
\hline ICG 13097 & Unknown & 96 & 80 & 88 \\
\hline ICG 13284 & Brazil & 92 & 98 & 95 \\
\hline ICG 13288 & Brazil & 98 & 94 & 96 \\
\hline ICG 13430 & Chad & 92 & 82 & 87 \\
\hline ICG 13513 & $\begin{array}{l}\text { Central } \\
\text { African } \\
\text { Republic }\end{array}$ & 90 & 98 & 94 \\
\hline ICG 13829 & Uganda & 90 & 78 & 84 \\
\hline ICG 14007 & $\begin{array}{l}\text { Central } \\
\text { African } \\
\text { Republic }\end{array}$ & 82 & 70 & 76 \\
\hline $\begin{array}{l}\text { ICG } 14696 \\
\text { var. hypogaea }\end{array}$ & Brazil & 90 & 92 & 91 \\
\hline ICG 956 & India & 86 & 72 & 79 \\
\hline ICG 1975 & Sudan & 96 & 74 & 85 \\
\hline ICG 2422 & India & 88 & 70 & 79 \\
\hline ICG 2506 & India & 96 & 76 & 86 \\
\hline ICG 2777 & India & 98 & 73 & 86 \\
\hline ICG 2925 & India & 86 & 71 & 79 \\
\hline ICG 3877 & India & 98 & 76 & 87 \\
\hline ICG 3987 & India & 100 & 76 & 88 \\
\hline ICG 4243 & Australia & 94 & 72 & 83 \\
\hline ICG 4250 & Senegal & 88 & 71 & 80 \\
\hline ICG 4331 & India & 96 & 76 & 86 \\
\hline
\end{tabular}

Table 1 continued

\begin{tabular}{|c|c|c|c|c|}
\hline \multirow[t]{2}{*}{ Identity } & \multirow[t]{2}{*}{ Origin } & \multicolumn{3}{|c|}{ Germination $(\%)$} \\
\hline & & $\begin{array}{l}1999 / 2000 \\
\text { post-rainy }\end{array}$ & $\begin{array}{l}2000 / 2001 \\
\text { post-rainy }\end{array}$ & Mean \\
\hline ICG 4738 & $\begin{array}{l}\text { United } \\
\text { Kingdom }\end{array}$ & 90 & 76 & 83 \\
\hline ICG 5163 & Brazil & 98 & 78 & 88 \\
\hline ICG 5233 & Israel & 90 & 70 & 80 \\
\hline ICG 6143 & USA & 82 & 76 & 79 \\
\hline ICG 6361 & India & 96 & 75 & 86 \\
\hline ICG 6515 & Israel & 100 & 72 & 86 \\
\hline ICG 6686 & China & 86 & 84 & 85 \\
\hline ICG 7458 & Nigeria & 92 & 82 & 87 \\
\hline ICG 7932 & South Africa & 86 & 71 & 79 \\
\hline ICG 8748 & Russia \& CIS & 100 & 82 & 91 \\
\hline ICG 8833 & USA & 92 & 70 & 81 \\
\hline ICG 8835 & USA & 94 & 70 & 82 \\
\hline ICG 9037 & Côte d'Ivoire & 98 & 70 & 84 \\
\hline ICG 9515 & Mozambique & 96 & 74 & 85 \\
\hline ICG 9556 & Mozambique & 92 & 72 & 82 \\
\hline ICG 9695 & India & 82 & 70 & 76 \\
\hline ICG 9873 & Zambia & 94 & 94 & 94 \\
\hline ICG 10105 & Chad & 86 & 82 & 84 \\
\hline ICG 10575 & Israel & 94 & 82 & 88 \\
\hline ICG 11109 & Taiwan & 98 & 80 & 89 \\
\hline ICG 11456 & India & 94 & 76 & 85 \\
\hline ICG 12360 & India & 100 & 83 & 92 \\
\hline ICG 13539 & Togo & 98 & 71 & 85 \\
\hline ICG 13724 & Niger & 88 & 70 & 79 \\
\hline \multicolumn{5}{|c|}{ var. peruviana } \\
\hline ICG 1709 & Peru & 96 & 94 & 95 \\
\hline ICG 1710 & Peru & 90 & 96 & 93 \\
\hline ICG 7293 & Peru & 94 & 92 & 93 \\
\hline ICG 10036 & Peru & 90 & 90 & 90 \\
\hline ICG 10037 & Peru & 82 & 100 & 91 \\
\hline ICG 10567 & Peru & 92 & 92 & 92 \\
\hline ICG 10911 & Peru & 86 & 82 & 84 \\
\hline ICG 10915 & Peru & 84 & 84 & 84 \\
\hline ICG 10945 & Peru & 88 & 96 & 92 \\
\hline ICG 11088 & Peru & 92 & 100 & 96 \\
\hline $\begin{array}{l}\text { ICG } 12112 \\
\text { var. vulgaris }\end{array}$ & Peru & 92 & 94 & 93 \\
\hline ICG 1364 & India & 86 & 80 & 83 \\
\hline ICG 1988 & Brazil & 96 & 82 & 89 \\
\hline ICG 2344 & USA & 88 & 78 & 83 \\
\hline ICG 4749 & Argentina & 84 & 82 & 83 \\
\hline
\end{tabular}


Table 1 continued

\begin{tabular}{|c|c|c|c|c|}
\hline \multirow[t]{2}{*}{ Identity } & \multirow[t]{2}{*}{ Origin } & \multicolumn{3}{|c|}{ Germination $(\%)$} \\
\hline & & $\begin{array}{l}1999 / 2000 \\
\text { post-rainy }\end{array}$ & $\begin{array}{l}2000 / 2001 \\
\text { post-rainy }\end{array}$ & Mean \\
\hline $\begin{array}{l}\text { ICG } 14966 \\
\text { Control }\end{array}$ & Unknown & 92 & 73 & 83 \\
\hline $\begin{array}{c}\text { Gangapuri } \\
\text { (ICG } \\
2738 \text { ) }\end{array}$ & India & 84 & 90 & 87 \\
\hline $\begin{array}{c}\text { ICGS } 44 \\
\quad(I C G \\
13941)\end{array}$ & India & 43 & 66 & 55 \\
\hline $\begin{array}{c}\text { ICGS } 76 \\
\text { (ICG } \\
13942)\end{array}$ & India & 50 & 42 & 46 \\
\hline $\begin{array}{c}\text { M-13 } \\
\text { (ICG } \\
156)\end{array}$ & India & 50 & 22 & 36 \\
\hline $\begin{array}{c}\text { Trial mean } \\
(1,708 \\
\text { entries })\end{array}$ & & 57.9 & 48.5 & 53.2 \\
\hline $\mathrm{SE} \pm$ & & 10.69 & 7.253 & 6.20 \\
\hline $\mathrm{CV}(\%)$ & & 32.33 & 18.46 & 14.5 \\
\hline $\begin{array}{l}\text { LSD } \\
\qquad(P=0.05)\end{array}$ & & 20.95 & 20.14 & 17.2 \\
\hline
\end{tabular}

percentage were recorded on a plot basis; number of primary branches, plant height, leaflet length and width, pods per plant, and pod yield per plant were recorded on five competitive plants; seeds per pod, and pod length and width were recorded on ten randomly selected mature pods; seed length and width were based on ten mature seeds; shelling percentage was on $200 \mathrm{~g}$ pods; and seed weight was of 100 randomly selected mature seeds. Oven-dried $\left(100^{\circ} \mathrm{C}, 16 \mathrm{~h}\right)$ bulked seed samples were used to determine oil and protein contents in both the seasons. Oil content was determined using a magnetic resonance spectrometer (Jambunathan et al. 1985), and data was corrected to uniform $50 \mathrm{~g} \mathrm{~kg}^{-1}$ seed moisture content. Nitrogen concentration was determined by Technicon Autoanalyser (Pulse Instrumentation Ltd., Saskatoon SK) and then multiplied by 5.46 to convert nitrogen into crude protein content (Singh and Jambunathan 1980).

Data were analyzed by the residual maximumlikelihood (REML) mixed model method with genotypes as random and environments (seasons) as fixed in GENSTAT 9.1 (Payne et al. 2006). The best linear unbiased predictors (BLUPs) were calculated for 15 agronomic and two quality traits. Homogeneity of variances in two seasons was tested by the Bartlett's test of homogeneity (Bartlett 1937). Meta-analysis of two seasons' data was performed when variances were heterogeneous. The components of variance due to the various botanical types as a group and individually and their interactions with season were also estimated for all traits to determine if the botanical types differed or interacted with environment. Also a comprehensive genotype-byenvironment analysis, considering all genotypes as one group, was done, and the variance components due to genotype $\left(\sigma_{\mathrm{g}}^{2}\right)$, genotype-by-environment $\left(\sigma_{\mathrm{ge}}^{2}\right)$, and residual variance $\left(\sigma_{\mathrm{e}}^{2}\right)$ and their standard errors were calculated.

A phenotypic distance matrix was created by calculating the differences between all pair of accessions using all the descriptors. The diversity index was calculated by averaging all the differences in the phenotypic values for each trait divided by its respective range (Johns et al. 1997).

The mean observations of all traits for each environment were standardized by subtracting from each observation the mean value of the character and dividing by its respective standard deviation, providing standardized values for each trait with an average of 0 and standard deviation of 1 . The standardized values were used for principal component analysis (PCA) using GENSTAT 9.1 (Payne et al. 2006). Cluster analysis was performed using scores of the first nine principal components (Ward 1963). Means and variances for quantitative traits in the different clusters were calculated. Differences for means among the clusters were tested using the NewmanKeuls procedure (Newman 1939; Keuls 1952) while the homogeneity of variances among the clusters was tested using Levene's test (Levene 1960).

\section{Results and discussion}

Analysis based on botanical varieties

REML analysis indicated that the effect of season was highly significant for all agronomic traits ( $P \leq 0.001-0.005)$, except for the number of primary branches. The effect of botanical variety was also highly significant $(P \leq 0.001-0.003)$ for all traits, 
except pod and seed width, and 100-seed weight. The season $\times$ botanical variety interaction was significant for nine traits $(P \leq 0.001-0.035)$, and nonsignificant for six traits (days to flowering, plant height, pod width, seed length, shelling percentage, and seed weight). In the 2001 rainy season, the effect of botanical variety was significant for five traits (leaf and pod length, seed width, plot yield, and shelling percentage) while in the 2001/2002 post-rainy season botanical variety was significant for all traits except for pod and seed width, shelling percentage, and seed weight (data not sown).

Estimates of components of variance for agronomic traits

Genotypic variance $\left(\sigma_{\mathrm{g}}^{2}\right)$ was significant for 15 traits in 2001 rainy season and for 13 traits in 2001/2002 post rainy-season (Table 2). In the combined analysis (meta analysis) genotypic variance was significant for primary branches, plant height, leaflet length, leaflet width, seed width, and 100-seed weight (Table 2). Genotype-by-environment $\left(\sigma_{\mathrm{ge}}^{2}\right)$ interaction was significant for all 15 traits.
Performance of cold-tolerant germplasm for agronomic traits

Accessions from fastigiata, aequatoriana, and peruviana (subsp. fastigiata) groups were compared with the control cultivar Gangapuri; accessions from vulgaris (subsp. fastigiata) were compared with control cultivar ICGS 44; and those belonging to hypogaea (subsp. hypogaea) were compared with with control cultivars ICGS 76 and M13. Table 3 lists the accessions with superior performance over their respective controls for various traits among different botanical varieties in the rainy and post-rainy seasons and across seasons. Of these, only 41 accessions from the five botanical varieties were significantly superior to their respective controls for 1-3 traits in the combined analysis. For example, 15 accessions were superior to the controls for pod yield (ICG\#10915, 10567, 1710, 11088, 10945, 12625, 7898, 11130, 6148, 6022, 7013, 7905, 7884, 9515, and 4992), 5 for faster seed emergence (ICG\#2422, 1364, 2344, 4749, and 1988), 1 for days to 50\% flowering (ICG 14966), 9 for oil (ICG\# 8833, 9695, 10575, 10036, 11203, 6340, 13513, 13430, and 14007) and 11 for protein (ICG\# 9556, 8835, 9515,

Table 2 Estimates of variance components due to genotype $\left(\sigma_{\mathrm{g}}^{2}\right)$ and genotype $\times$ environment $\left(\sigma_{\mathrm{ge}}^{2}\right)$ and their standard

2001/2002 post-rainy seasons and combined analysis in coldtolerant peanut germplasm evaluated at ICRISAT, Patancheru, India errors (SE) for 15 quantitative traits in the 2001 rainy and

\begin{tabular}{|c|c|c|c|c|c|c|c|c|}
\hline \multirow[t]{2}{*}{ Trait } & \multicolumn{2}{|c|}{2001 rainy season } & \multicolumn{2}{|c|}{$\begin{array}{l}2001-02 \text { post-rainy } \\
\text { season }\end{array}$} & \multicolumn{4}{|c|}{ Combined analysis } \\
\hline & $\sigma_{\mathrm{g}}^{2}$ & SE & $\sigma_{\mathrm{g}}^{2}$ & SE & $\sigma_{\mathrm{g}}^{2}$ & SE & $\sigma_{\mathrm{ge}}^{2}$ & SE \\
\hline Time to $50 \%$ emergence (days) & 0.295 & 0.060 & 0.176 & 0.049 & 0.025 & 0.041 & 0.289 & 0.056 \\
\hline Time to $50 \%$ flowering (days) & 8.329 & 0.970 & 2.547 & 0.534 & 0.046 & 0.622 & 6.512 & 0.854 \\
\hline Primary branch (no.) & 0.338 & 0.050 & 0.024 & 0.147 & 0.019 & 0.062 & 0.295 & 0.077 \\
\hline Plant height $(\mathrm{cm})$ & 37.990 & 4.820 & 9.742 & 1.226 & 4.885 & 2.313 & 21.272 & 2.279 \\
\hline Leaflet length (mm) & 31.590 & 4.260 & 24.580 & 3.104 & 6.620 & 3.330 & 30.240 & 3.920 \\
\hline Leaflet width $(\mathrm{mm})$ & 1.705 & 0.310 & 2.188 & 0.328 & 0.630 & 0.293 & 2.173 & 0.343 \\
\hline Pods per plant (no.) & 3.775 & 0.820 & 3.710 & 1.250 & $<0.001$ & $<0.01$ & 4.850 & 0.770 \\
\hline Pod length (mm) & 15.110 & 2.120 & 19.749 & 2.492 & 2.470 & 1.800 & 16.660 & 2.260 \\
\hline Pod width (mm) & 0.818 & 0.110 & 2.340 & 1.720 & $<0.001$ & $<0.01$ & 1.733 & 0.238 \\
\hline Seed length (mm) & 1.822 & 0.260 & 1.811 & 0.237 & 0.215 & 0.185 & 1.733 & 0.238 \\
\hline Seed width (mm) & 0.175 & 0.040 & 0.192 & 0.035 & 0.056 & 0.025 & 0.124 & 0.029 \\
\hline Pod yield per plant $(\mathrm{g})$ & 2.592 & 0.670 & 10.440 & 2.070 & 0.340 & 0.830 & 5.390 & 1.230 \\
\hline Pod yield per plot $\left(\mathrm{kg} \mathrm{h}^{-1}\right)$ & 29123.000 & 5135.000 & 256426.000 & 35076.000 & 30170.000 & 16270.000 & 155732.000 & 19871.000 \\
\hline Shelling percentage & 15.120 & 2.590 & 11.430 & 1.960 & 1.740 & 1.560 & 11.560 & 2.070 \\
\hline 100 seed weight $(\mathrm{g})$ & 22.610 & 3.570 & 46.470 & 6.060 & 6.580 & 3.240 & 26.450 & 3.840 \\
\hline
\end{tabular}


Table 3 Accessions better or significantly better for various traits compared with their respective control cultivars in the 2001 rainy and 2001-2002 post-rainy seasons and combined analysis

\begin{tabular}{|c|c|c|c|}
\hline Trait & 2001 rainy season & 2001-2002 post-rainy season & Combined \\
\hline \multirow[t]{5}{*}{ Emergence } & $\begin{array}{l}\text { FST }^{\text {a }}: 1899,6725,7005,7812,3125 \\
\quad 13097,115, \text { and } 3219^{\text {f }}\end{array}$ & $\mathrm{AEQ}^{\mathrm{e}}: 7898$ & AEQ: 12625 \\
\hline & $\mathrm{PRU}^{\mathrm{b}}: 10911$ & $\begin{array}{l}\text { FST: } 6221,5964,9929,389, \text { and } \\
\quad 4992\end{array}$ & FST: 7355, 6725, 115, 4992, 389 \\
\hline & VUL $^{c}: 1988,4749$ and 2343 & $\begin{array}{l}\text { HYP: } 11456^{*}, 6686^{*}, 13724^{*}, 9556^{*} \\
\text { and } 9515^{*}\end{array}$ & HYP: $2422^{*}, 6686,7932,9556,7458$, \\
\hline & $\begin{array}{l}\mathrm{HYP}^{\mathrm{d}}: 2925^{*}, 6686^{*}, 7458^{*}, 1975^{*}, \\
\text { 2506* and } 956^{*}\end{array}$ & $\begin{array}{l}\text { PRU: } 11088,10567,1709,10911 \\
\quad \text { and } 7293\end{array}$ & PRU: 10567, 1709, 7293, 10911 \\
\hline & $\begin{array}{l}\text { VUL: } 1988 *, 2344,14966,4749 \text {, } \\
1364\end{array}$ & VUL: $1364 *, 2344 *, 4749^{*}, 1988^{*}$ & \\
\hline \multirow[t]{3}{*}{$\begin{array}{l}\text { Days to } \\
\text { flowering }\end{array}$} & $\begin{array}{l}\text { FST: } 7978^{*}, 4890,7352,6340,10616 \text {, } \\
\text { 10402, }\end{array}$ & FST: $1899 *, 8570,7285,1158,5964$ & FST: 7978, 7285, 10402, 1158, 1899 \\
\hline & $3125,115,3219,6570,8514,12498$ & HYP: $1975,6143,6515$ & HYP: $6515,1975,956,9556,6143$ \\
\hline & VUL: 1988, 4749, 2344 & VUL: 14966,4749 & VUL: $14966^{*}$ \\
\hline \multirow{4}{*}{$\begin{array}{l}\text { Pod yield } \\
\text { per plot }\end{array}$} & AEQ : $12625^{*}$ & AEQ $12553^{*}, 12625^{*}, 7898^{*}, 12719$ & AEQ: $12625^{*}, 7898^{*}$ \\
\hline & $\begin{array}{l}\text { FST: } 13284^{*}, 2039^{*}, 13513^{*} \text { and } \\
\quad 1824^{*}\end{array}$ & $\begin{array}{l}\text { FST: } 10595^{*}, 6148^{*}, 6022^{*}, 7013^{*} \\
\text { 7884* } \\
\text { (9905* and } 4992^{*}\end{array}$ & $\begin{array}{l}\text { FST: } 11130^{*}, 6148^{*}, 7013^{*}, 6022^{*}, \\
\text { 7905*, 7884*, 4992* }\end{array}$ \\
\hline & HYP: $6686^{*}$ & $\begin{array}{l}\text { PRU: } 10037 *, 10567 *, 1717^{*}, \\
\quad 11088^{*} \text { and } 10945^{*}\end{array}$ & HYP: $9515^{*}, 1975,9556,956,13539$ \\
\hline & $\begin{array}{l}\text { PRU: } 10036^{*}, 10567^{*}, 1710^{*}, 1709^{*} \\
10915^{*}, 10945^{*}, 11088^{*}\end{array}$ & & $\begin{array}{l}\text { PRU: } 10915^{*}, 10567^{*}, 1710^{*}, 11088^{*}, \\
\quad 10945^{*}\end{array}$ \\
\hline \multirow{4}{*}{$\begin{array}{l}\text { Pods per } \\
\text { plant }\end{array}$} & AEQ 12719,12625 & AEQ: $12719,7898,12625,12553$ & AEQ: $7898,12719,12553,12625$ \\
\hline & $\begin{array}{l}\text { FST: } 4087^{*}, 1824^{*}, 11130 * \text { and } \\
\quad 8360^{*}\end{array}$ & $\begin{array}{l}\text { FST: } 11130,10616,2145,7929, \\
\quad 5609\end{array}$ & FST: $1899,7929,13049,5609,11130$ \\
\hline & $\begin{array}{l}\text { HYP: } 6686^{*}, 11109,3877,4431 \\
\quad 5163,1975\end{array}$ & HYP: 3987, 7932, 13539, 9037 & HYP: 9556, 13539, 9037 \\
\hline & PRU: 10036, 1710, 7293, 1709, 10567 & $\begin{array}{l}\text { PRU: } 10036,10945,10911,10915 \text {, } \\
\quad 11088\end{array}$ & $\begin{array}{l}\text { PRU: } 10036,10911,11088,10915 \text {, } \\
\quad 10567\end{array}$ \\
\hline \multirow{5}{*}{$\begin{array}{l}\text { Yield per } \\
\text { plant }(\mathrm{g})\end{array}$} & AEQ: 12719,12625 & AEQ: $12625^{*}, 12553^{*}, 7898^{*}$ & AEQ: $12625^{*}, 7898^{*}, 12719,12553$ \\
\hline & FST: $13513^{*}, 1824^{*}, 13049^{*}$ & FST: $4992 *$ and $6022^{*}$ & FST: $6022 *, 12963,13049,6340,4992$ \\
\hline & HYP: 4331, 1975, 7932, 6686, 11109 & HYP: 13539 & HYP: 7932, 13539 \\
\hline & PRU: 7293, 10567, 1710, 11088 & $\begin{array}{l}\text { PRU: } 12112^{*}, 1710^{*}, 10915^{*} \\
10945^{*}, 10911^{*} \text { and } 11088^{*}\end{array}$ & $\begin{array}{l}\text { PRU: } 10911^{*}, 10567^{*}, 12112^{*} \\
\text { 10915*, 10945*, 1710* } 11088^{*}\end{array}$ \\
\hline & VUL: 2344,14896 & & VUL: 2344 \\
\hline \multirow[t]{3}{*}{$\begin{array}{l}\text { Shelling } \\
\text { percentage }\end{array}$} & $\begin{array}{l}\text { FST: } 13829,1796,14696,1824 \text { and } \\
\quad 2159\end{array}$ & $\begin{array}{l}\text { FST: } 12498,11203,10549,397 \text { and } \\
\quad 3510\end{array}$ & $\begin{array}{l}\text { FST: } 6570,12498,13829,14696 \text {, } \\
\quad 11203\end{array}$ \\
\hline & PRU: 1710 & HYP: 1975 & \\
\hline & & VUL: 10037 & \\
\hline \multirow{5}{*}{$\begin{array}{r}\text { 100-Seed } \\
\text { weight }\end{array}$} & AEQ: 12625 & AEQ: $12625,12553,7898$ & AEQ: 7898,12625 \\
\hline & FST: $8485^{*}, 457,6220,1274,1824$ & $\begin{array}{l}\text { FST: } 7013^{*}, 14007^{*}, 6022^{*} \text { and } \\
\quad 6148^{*}\end{array}$ & FST: $8485^{*}, 14007^{*}, 6022^{*}, 6148^{*}$ \\
\hline & PRU: 1709, 11088, 12112, 1710 & HYP: 8748 & $\begin{array}{l}\text { PRU: } 12112^{*}, 1710^{*}, 10567,10037 \text {, } \\
\quad 11088\end{array}$ \\
\hline & VUL: 14966 & PRU: $12112^{*}$ and $1710^{*}$ & VUL: 14966 \\
\hline & & & VUL: 14966, 1364 \\
\hline
\end{tabular}


Table 3 continued

\begin{tabular}{llll}
\hline Trait & 2001 rainy season & 2001-2002 post-rainy season & Combined \\
\hline $\begin{array}{l}\text { Oil } \\
\text { content }^{\mathrm{f}}\end{array}$ & AEQ: $12553,12719,12625$ & AEQ: 7898, 12625, 12553, 12719 & AEQ: 12625 \\
& FST: $14007^{*}, 13513^{*}, 13430^{*}$ & FST: 7777, 282, 10900, 12743, & FST: 11203*, 6340*, 14007*, 13513*, \\
& & 12665 & $13430^{*}$ \\
& HYP: $10575^{*}, 9695,8833$ & HYP: $3987,2925,2506,10575,4250$ & HYP: $8833^{*}, 9695^{*}, 10575^{*}, 9873$, \\
& & & 6686,11109
\end{tabular}

$\overline{{ }^{\mathrm{a}} \mathrm{FST}}=$ Fastigata,${ }^{\mathrm{b}} \mathrm{PRU}=$ Peruviana,${ }^{\mathrm{c}}$ VUL $=$ Vulgaris,${ }^{\mathrm{d}}$ HYP $=$ Hypogaea,${ }^{\mathrm{e}}$ AEQ $=$ Aequatoriana,${ }^{\mathrm{f}}$ Analysis carried out at entry level only

* Accessions significantly better over their respective controls

$10105,4331,1256,1975,7355,398,8485$, and 1384) contents, 3 for pod yield and seed weight (ICG\#1710, 6022, and 6148), and 1 each for pod yield and protein content (ICG 9515), seed weight and protein content (ICG 8485), and for seed weight and oil content (ICG 14007). However, only ICG\# 12625, 10567, 1710 , 10945 , and 11088 were significantly superior for pod yield in both seasons. The variable performance of many accessions was mainly due to the significant genotype-by-environment interaction observed for most traits (Table 2).

Cluster composition and variation for

morphological traits among clusters

PCAs based on the first nine principal components accounted for $79 \%$ of the total variation and resulted in four distinct clusters (Table 4). Cluster 1 comprised 23 accessions dominated by peruviana $(47.8 \%)$ and fastigiata (30.4\%) types. A majority of accessions in this cluster have erect growth habit, sequential branching, no stem pigmentation, leaflets almost glabrous on surfaces, peg pigmentation, pods with slight constriction and reticulation, and had 3-2-4-1/3-2-1-4/3-4-2-1 seeds per pod (more three-seeded than the other type of pods). Although there were five primary seed colors most of the accessions had tan-colored seeds. Of the 26 accessions included in cluster 2, $81 \%$ were hypogaea types, with most of the accessions having procumbent growth habit, alternate branching, no pigmentation on the stem (but pigmentation on pegs), subglabrous hairs in one or two rows along main axis, and green and glabrous leaflets. Most of the accessions have moderate pod beak and constriction, slight reticulation, and 2-1 seeds per pod (a high frequency of more two-seeded pods). The predominant seed color in this cluster was tan but the cluster included eight primary seed colors. In cluster 3, 54.3\% of the accessions belonged to fastigiata and $34.3 \%$ to hypogaea. The cluster was characterized by erect growth with sequential branching, stem and peg pigmentation, subglabrous hairs on the main axis, light green glabrous leaflets, pods with slight beak, constriction and reticulation, and 3-2-4-1/ 3-2-1-4/3-4-2-1 seeds per pod. Red- and tan-colored seeds were predominant, although nine primary seed colors were recorded. Cluster 4 accessions were predominantly from fastigiata (95\%), mostly with erect growth habit, sequential branching, stem and peg pigmentation, subglabrous hairs on the main axis, light-green glabrous leaflets, slight pod beak and constriction, and 3-2-4-1/3-2-1-4/3-4-2-1 seeds per pod. Red seed color was predominant, although eight primary seed colors were observed.

Variation for agronomic traits among clusters

In the rainy season the four clusters differed significantly for all traits except for pods per plant and pod 
Table 4 Distribution of cold-tolerant peanut accessions and control cultivars in four clusters delineated by cluster analysis based on scores of nine principal components

\begin{tabular}{|c|c|c|}
\hline Cluster & $\begin{array}{l}\text { Botanical } \\
\text { varieties }\end{array}$ & Accessions (ICG number) \\
\hline \multirow[t]{4}{*}{1} & aequatoriana & ICGs $7898,12553,12625$, and 12719 \\
\hline & fastigiata & ICGs $4992,6022,6148,6340,7013,7884$, and 14007 \\
\hline & hypogaea & ICG 7932 \\
\hline & peruviana & ICGs 1709, 1710, 7293, 10036, 10037, 10567, 10911, 10915, 10945, 11088, and 12112 \\
\hline \multirow[t]{3}{*}{2} & fastigiata & ICGs $1683,10075,10554$, and 11203 \\
\hline & hypogaea & $\begin{array}{l}\text { ICGs } 956,1975,2422,2506,2777,2925,3877,3987,4331,5163,6143,6361,8748,8833,8835,9037 \text {, } \\
\quad 9556,9695,10105,10575 \text {, and } 11109\end{array}$ \\
\hline & vulgaris & ICG 2344 \\
\hline \multirow[t]{3}{*}{3} & fastigiata & $\begin{array}{l}\text { ICGs } 457,1274,1796,1824,2158,2159,3510,4670,4890,5609,5964,6220,6565,8485,9141,9144 \text {, } \\
9929,10481 \text {, and } 10595\end{array}$ \\
\hline & hypogaea & M 13, ICGs 4243, 4250, 4738, 6686, 9515, 9873, 11456, 12360, 13539, and 13724, and ICGS 76 \\
\hline & vulgaris & ICGs $1364,4749,14966$ and ICGS 44 \\
\hline \multirow[t]{3}{*}{4} & fastigiata & $\begin{array}{l}\text { ICGs } 115,282,318,376,389,397,398,445,1158,1256,1298,1384,1399,1899,1908,2039,2057,2145, \\
\quad 3125,3219,3477,3726,3779,4087,4788,5094,5475,6203,6221,6421,6570,6706,6725,6878,6888, \\
7005,7285,7352,7355,7777,7812,7905,7929,7978,8360,8514,8517,8570,10092,10371,10402, \\
\quad 10495,10519,10549,10566,10616,10788,10900,11130,11605,12498,12564,12665,12743,12963, \\
\text { 13049, 13097, 13284, 13288, 13430, 13513, 13829, and 14696, and Gangapuri }\end{array}$ \\
\hline & hypogaea & 5233,6515 , and 7458 \\
\hline & vulgaris & 1988 \\
\hline
\end{tabular}

width (Table 5). Cluster 2 and 4 accessions emerged faster and flowered earlier. Accessions in cluster 4 were taller, had large leaflets, and high seed protein content. Accessions in cluster 3 were shorter and had small leaflets, more primary branches, the highest seed yield, a high shelling percentage, and large seed size. Accessions in cluster 2 had the highest seed oil content. In the post-rainy season, differences were significant among four clusters for 17 traits. Cluster 1 and 4 accessions emerged faster and flowered earlier. Plants in cluster 1 accessions were taller, had larger leaflets, highest seed yield, large pod and seed size, and high seed oil content.

Heterogeneity of variances for various traits

Variance for 9 agronomic traits in the 2001 rainy and 8 traits in the 2001/2002 post-rainy seasons were heterogeneous (Table 6). In the rainy season, cluster 1 accessions had higher variances for plant height and leaflet length and cluster 3 accessions for pod yield per plot, 100-seed weight, and seed protein content. Higher variances for accessions in cluster 1 for pod length, in cluster 2 for pod yield and 100-seed weight, and in cluster 3 for pods per plant occurred in the post-rainy season.

Phenotypic diversity index among clusters

The clusters differed in terms of the biological status (whether landraces, breeding lines or cultivars) of the accessions involved in minimum and maximum phenotypic diversity indices (Table 7). Accessions with the least difference in phenotypic diversity index in the entire set were ICG 13288 (a landrace from Brazil) and ICG 10519 (an advanced cultivar from Australia). The accessions with the minimum diversity index in each cluster were two landraces from Peru (ICG 10945 and ICG 11088) in cluster 1, breeding lines (ICG 2506 and ICG 2925) from India in cluster 2, a landrace (ICG 6220) from Brazil and an advanced cultivar (ICG 9144) from Syria in cluster 3, and an accession with unknown origin (ICG 1298) and Indian cultivar Gangapuri (ICG 2738) in cluster 4 .

The maximum phenotypic diversity index in the entire set was between ICG 12112 (landrace from 
Table 5 Mean for agronomic traits in different clusters of cold-tolerant peanut germplasm accessions in the 2001 rainy and 20012002 post-rainy seasons at ICRISAT Center, Patancheru, India

\begin{tabular}{|c|c|c|c|c|}
\hline Trait & Cluster 1 & Cluster 2 & Cluster 3 & Cluster 4 \\
\hline \multicolumn{5}{|l|}{ Rainy season } \\
\hline Time to emergence (days) & $8.0 \mathrm{~b}$ & $8.0 \mathrm{~b}$ & $8.4 \mathrm{a}$ & $8.4 \mathrm{a}$ \\
\hline Time to $50 \%$ flowering (days) & $21.1 \mathrm{~b}$ & $19.8 \mathrm{c}$ & $25.4 \mathrm{a}$ & $19.9 \mathrm{c}$ \\
\hline Primary branch (no.) & $4.6 b$ & $4.4 \mathrm{~b}$ & $5.3 \mathrm{a}$ & $4.4 b$ \\
\hline Plant height $(\mathrm{cm})$ & $28.6 \mathrm{a}$ & $28.8 \mathrm{a}$ & $18.7 \mathrm{~b}$ & $30.6 \mathrm{a}$ \\
\hline Leaflet length (mm) & $54.2 \mathrm{a}$ & $53.5 \mathrm{a}$ & $44.7 b$ & $55.2 \mathrm{a}$ \\
\hline Leaflet width $(\mathrm{mm})$ & $23.0 \mathrm{a}$ & $22.9 \mathrm{a}$ & $21.5 b$ & $23.2 \mathrm{a}$ \\
\hline Pods per plant (no.) & $10.1 \mathrm{a}$ & $10.7 \mathrm{a}$ & $10.3 \mathrm{a}$ & $10.7 \mathrm{a}$ \\
\hline Pod length (mm) & $33.3 \mathrm{a}$ & $33.0 \mathrm{a}$ & $31.0 \mathrm{~b}$ & $33.5 \mathrm{a}$ \\
\hline Pod width (mm) & $12.8 \mathrm{a}$ & $12.8 \mathrm{a}$ & $12.5 \mathrm{a}$ & $12.8 \mathrm{a}$ \\
\hline Seed length (mm) & $14.2 \mathrm{ba}$ & $13.9 \mathrm{~b}$ & $14.7 \mathrm{a}$ & $14.0 \mathrm{ba}$ \\
\hline Seed width (mm) & $7.7 \mathrm{~b}$ & $7.6 \mathrm{~b}$ & $7.9 \mathrm{a}$ & $7.6 \mathrm{~b}$ \\
\hline Pod yield per plant (g) & $7.8 \mathrm{a}$ & $7.9 \mathrm{a}$ & $7.1 \mathrm{~b}$ & $7.8 \mathrm{a}$ \\
\hline Pod yield per plot (kg) & $529.2 b$ & $517.2 \mathrm{~b}$ & $617.9 \mathrm{a}$ & $492.8 b$ \\
\hline Shelling (\%) & $58.1 \mathrm{~b}$ & $59.8 \mathrm{ba}$ & $61.1 \mathrm{a}$ & $59.0 \mathrm{~b}$ \\
\hline 100-seed weight $(\mathrm{g})$ & $32.6 b$ & $32.1 \mathrm{~b}$ & $35.0 \mathrm{a}$ & $32.3 b$ \\
\hline Oil content $(\%)$ & $47.7 \mathrm{ba}$ & $48.3 \mathrm{a}$ & $47.1 \mathrm{bc}$ & $46.5 \mathrm{c}$ \\
\hline Protein content $(\%)$ & $25.3 b$ & $23.7 \mathrm{c}$ & $25.5 b$ & $27.4 \mathrm{a}$ \\
\hline \multicolumn{5}{|l|}{ Post-rainy season } \\
\hline Time to emergence (days) & $12.0 \mathrm{c}$ & $12.8 \mathrm{a}$ & $12.4 \mathrm{~b}$ & $12.1 \mathrm{c}$ \\
\hline Time to $50 \%$ flowering (days) & $48.3 b$ & $50.0 \mathrm{a}$ & $48.0 \mathrm{~b}$ & $47.6 \mathrm{~b}$ \\
\hline Primary branch (no.) & $4.5 b$ & $4.7 \mathrm{a}$ & $4.6 \mathrm{~b}$ & $4.5 b$ \\
\hline Plant height $(\mathrm{cm})$ & $22.1 \mathrm{a}$ & $13.0 \mathrm{~d}$ & $16.5 \mathrm{c}$ & $18.1 \mathrm{~b}$ \\
\hline Leaflet length (mm) & $57.5 \mathrm{a}$ & $41.9 \mathrm{c}$ & $46.0 \mathrm{~b}$ & $47.4 \mathrm{~b}$ \\
\hline Leaflet width $(\mathrm{mm})$ & $24.3 \mathrm{a}$ & $19.8 \mathrm{c}$ & $20.7 b$ & $21.2 b$ \\
\hline Pods per plant (no.) & $16.2 b$ & $17.3 \mathrm{a}$ & $16.0 \mathrm{~b}$ & $15.0 \mathrm{c}$ \\
\hline Pod length (mm) & $40.2 \mathrm{a}$ & $33.3 b$ & $34.6 b$ & $35.2 b$ \\
\hline Pod width (mm) & $14.3 \mathrm{a}$ & $13.6 \mathrm{~b}$ & $13.8 \mathrm{~b}$ & $13.8 \mathrm{~b}$ \\
\hline Seed length (mm) & $14.5 \mathrm{a}$ & $13.7 \mathrm{~b}$ & $13.5 b$ & $12.7 \mathrm{c}$ \\
\hline Seed width (mm) & $8.3 \mathrm{a}$ & $8.0 \mathrm{~b}$ & $8.3 \mathrm{a}$ & $8.2 \mathrm{a}$ \\
\hline Pod yield per plant (g) & $22.3 \mathrm{a}$ & $17.8 \mathrm{~b}$ & $17.5 b$ & $15.4 \mathrm{c}$ \\
\hline Pod yield per plot (kg) & $2562.7 \mathrm{a}$ & $1745.4 b$ & $1688.4 b$ & $1360.2 \mathrm{c}$ \\
\hline Shelling (\%) & $65.8 b$ & $66.1 \mathrm{~b}$ & $68.0 \mathrm{a}$ & $68.4 \mathrm{a}$ \\
\hline 100-seed weight $(\mathrm{g})$ & $53.9 \mathrm{a}$ & $44.3 \mathrm{c}$ & $48.0 \mathrm{~b}$ & $46.7 \mathrm{cb}$ \\
\hline Oil content $(\%)$ & $49.6 \mathrm{a}$ & $48.9 \mathrm{ba}$ & $47.8 \mathrm{ba}$ & $47.4 \mathrm{c}$ \\
\hline Protein content (\%) & $23.6 b$ & $23.2 b$ & $24.6 \mathrm{ba}$ & $25.3 \mathrm{a}$ \\
\hline
\end{tabular}

Differences between means of different clusters were tested using the Newman-Keuls test

Means followed by the same letter are not significantly different at $P=0.05$

Peru) and ICG 156 (cultivar from India) while accessions with maximum diversity in individual clusters were ICG 12112 (landrace from Peru) and ICG 7932 (advance line from South Africa) in cluster 1, ICG 10075 (landrace from Peru) and ICG
9037 (landrace from Côte d'Ivoire) in cluster 2, ICG 10595 (landrace from Brazil) and ICG 13942 (cultivar from India) in cluster 3, and ICG 1908 (breeding line from India) and ICG 7352 (land race from Peru) in cluster 4. 
Table 6 Traits with heterogeneous variances in four clusters for various agronomic traits in cold-tolerant peanut germplasm accessions, 2001-rainy and 2001-2002 post-rainy seasons, at ICRISAT, Patancheru, India

\begin{tabular}{|c|c|c|c|c|c|c|c|}
\hline Trait & Entire & Cluster 1 & Cluster 2 & Cluster 3 & Cluster 4 & $F$ value & $P^{\mathrm{a}}$ \\
\hline \multicolumn{8}{|l|}{ Rainy season } \\
\hline Time to $50 \%$ emergence (days) & 0.2 & 0.2 & 0.1 & 0.2 & 0.1 & 4.48 & 0.0048 \\
\hline Primary branch (no.) & 0.2 & 0.2 & 0.1 & 0.2 & 0.1 & 3.99 & 0.0089 \\
\hline Plant height $(\mathrm{cm})$ & 34.5 & 27.4 & 14.5 & 11.4 & 8.7 & 3.93 & 0.0098 \\
\hline Leaflet length (mm) & 28.9 & 26.3 & 7.5 & 7.7 & 10.8 & 4.65 & 0.0038 \\
\hline Leaflet width (mm) & 1.2 & 1.3 & 0.5 & 0.2 & 0.9 & 4.74 & 0.0034 \\
\hline Pods per plant (no.) & 2.2 & 1.6 & 1 & 3.1 & 2.4 & 2.75 & 0.0449 \\
\hline Pod yield per plot(kg) & 22725.9 & 23830.5 & 11155.8 & 43158 & 13063.4 & 4.55 & 0.0043 \\
\hline 100-seed weight $(\mathrm{g})$ & 17.3 & 16.3 & 11.6 & 37.9 & 8.3 & 10.25 & $<.0001$ \\
\hline Protein content $(\%)$ & 5.6 & 2.6 & 5.7 & 6.8 & 2 & 5.37 & 0.0015 \\
\hline \multicolumn{8}{|l|}{ Post-rainy season } \\
\hline Pods per plant (no.) & 4.1 & 4.3 & 4 & 5.4 & 2.1 & 3.37 & 0.0200 \\
\hline Pod length (mm) & 18.9 & 39.9 & 24.1 & 9.4 & 7.4 & 7.39 & 0.0001 \\
\hline Seed length (mm) & 1.8 & 2.2 & 3.1 & 1.6 & 0.5 & 8.87 & $<.0001$ \\
\hline Seed width (mm) & 0.1 & 0.1 & 0.1 & 0.1 & 0.1 & 2.96 & 0.0341 \\
\hline Pod yield per plant (g) & 14.1 & 10.6 & 15.2 & 15 & 3.9 & 4.98 & 0.0025 \\
\hline Pod yield per plot (kg) & 390860.7 & 334815.9 & 401850.4 & 392666.8 & 80495.8 & 5.54 & 0.0012 \\
\hline Shelling (\%) & 6.8 & 5.5 & 14.5 & 5.7 & 3 & 7.56 & $<.0001$ \\
\hline 100-seed weight $(\mathrm{g})$ & 37.1 & 61 & 66.3 & 26 & 10.7 & 6.63 & 0.0003 \\
\hline
\end{tabular}

Variances were tested using Levene's test

a $P=$ probability at 0.5

Table 7 Phenotypic diversity in the cold-tolerant peanut germplasm accession in the entire set and in different clusters

\section{Entire set}

Phenotypic diversity

Mean phenotypic diversity index

0.188

Minimum phenotypic diversity index between ICG10519 and ICG 13288

0.062

Maximum phenotypic diversity index between ICG 12112 and ICG 156

0.408

Cluster 1

Mean phenotypic diversity index

0.277

Minimum phenotypic diversity index between ICG 10945 and ICG 11088

0.145

Maximum phenotypic diversity index between ICG 12112 and ICG 7932

0.437

Cluster 2

Mean phenotypic diversity index

0.261

0.138

Minimum phenotypic diversity index between ICG 2506 and ICG 2925

0.517

Cluster 3

Mean phenotypic diversity index

0.255

0.112

0.450

Maximum phenotypic diversity index between ICG 10595 and ICG 13942

Cluster 4

Mean phenotypic diversity index

0.209

0.101

Minimum phenotypic diversity index ICG 1298 and ICG 2738

0.410 
Botanical varieties and cold tolerance

Differences for chilling injury among botanical types of peanut have been reported. Sellschop and Salmon (1928) found Valencia and Spanish types highly sensitive while Virginia bunch type had exceptional hardiness. Bell et al. (1991) reported a positive association between rate of emergence and mean daily temperature $\left(17.8-23.2^{\circ} \mathrm{C}\right)$ in 16 peanut cultivars, indicating that air temperatures were always lower than those required for good germination. They however reported no significant differences $(P<0.05)$ in coefficients of temperature sensitivity either between cultivars of the same botanical type or between different botanical types. All cultivars used in their study had similar base temperature $\left(T_{\mathrm{b}}\right)$ values for emergence $\left(13.2^{\circ} \mathrm{C}\right)$. However, we found differences between accessions (irrespective of botanical type) in terms of their cold tolerance at emergence under lower temperatures $\left(12^{\circ} \mathrm{C}\right)$ under laboratory conditions (Upadhyaya et al. 2001), indicating that cold-tolerant accessions identified in this study captured greater diversity for base-temperature tolerance at germination.

The cold-tolerant accessions reported in this study had substantial diversity for most agronomic traits and thus should be good sources to use in breeding programs for developing peanut cultivar that germinate at lower temperatures. It will also be interesting to study the reaction of these cold-tolerant accessions at various growth stages at which peanut is vulnerable to cold injury. Some of the identified accessions have good agronomic potential, and hence their use in breeding programs will not adversely affect exploitation of additive genetic variance in a self-pollinated crop such as peanut.

Open Access This article is distributed under the terms of the Creative Commons Attribution Noncommercial License which permits any noncommercial use, distribution, and reproduction in any medium, provided the original author(s) and source are credited.

\section{References}

Agriculture Canada (1984) Modified description of variety. Peanut (Arachis hypogaea L.), variety OAC Garroy. Licensed no. 2439

Agriculture Canada (1989) Description of variety. Peanut (Arachis hypogaea, L.), variety OAC Ruby. Registration no. 1-82. Agriculture Canada, Ottawa
Bartlett MS (1937) Some examples of statistical methods of research in agriculture and applied biology. J R Stat Soc 4:137-170

Bell MJ, Shorter R, Mayer R (1991) Cultivar and environmental effects on growth and development of peanuts (Arachis hypogaea L). 1. Emergence and flowering. Field Crops Res 27:17-33

Bell MJ, Roy RC, Tollenaar M, Michaels TE (1994a) Importance of variation in chilling tolerance for peanut genotypic adaptation to cool short-season environments. Crop Sci 34:1030-1039

Bell MJ, Gillespie TJ, Roy RC, Michaels TE, Tollenaar M (1994b) Peanut leaf photosynthetic activity in cool field environments. Crop Sci 34:1023-1029

Benedict CR, Ketring DL (1972) Nuclear gene affecting greening in virescent peanut leaves. Plant Physiol 49:974976

Bhagat NR, Ahmad T, Lalwani HB, Patra GJ, Acharya D (1988) Screening of bunch peanut (Arachis hypogaea L. ssp. fastigiata) germplasm for cold tolerance in India. Trop Agric (Trinidad) 65:109-112

Bhagat NR, Dayal D, Acharya D (1992) Performance of Spanish peanuts during winter- summer season at two locations in India. Trop Agric (Trinidad) 69:93-95

Bunting AH, Gibbons RW, Wynne JC (1985) Peanut (Arachis hypogaea L.). In: Summerfield RJ, Roberts EH (eds) Grain legume crops. Collins, London, pp 747-800

Dan NT, Hong NX (1996) Collaborative research in Vietnam: needs and opportunities. In Gowda CLL, Ramakrishna A (eds) Summary proceedings of CLAN country coordinators' steering committee meeting, 4-6 Dec. 1995, ICRISAT Patancheru, India, pp 56-58

Ellis RS, Hong TD, Roberts EH (1985) The conduct of seed germination tests. Handbook of seed technology for genebanks. Vol. 1. Principles and methodology. IBPGR Rome, Italy, pp 94-198

Fu JR, Lu XH, Chen RZ, Zang BZ, Liu ZS, Lee ZS, Cai DY (1988) Osmoconditioning of peanut (Arachis hypogaea L.) seeds with PEG to improve vigour and some biochemical activities. Seed Sci Technol (Switzerland) 16:197-212

Golombok SD, Johanson C (1997) Effect of soil temperature on vegetative and reproductive growth and development in three Spanish genotypes of peanut (Arachis hypogaea). Peanut Sci 24:67-72

IBPGR and ICRISAT (1992) Descriptors for groundnut. International Board for Plant Genetic Resources, Rome, Italy; International Crops Research Institute for the SemiArid Tropics, Patancheru, India, $125 \mathrm{pp}$

Jambunathan R, Raju SM, Barde P (1985) Analysis of oil contents of groundnut by nuclear magnetic resonance spectrometry. J Sci Food Agric 36:162-166

Johns MA, Skroach PW, Nienhuis J, Hinrichsen P, Bascur G, Munoz-Schick C (1997) Gene pool classification of common bean landraces from Chile based on RAPD and morphological data. Crop Sci 37:605-613

Ketring DL (1984) Temperature effects on vegetative and reproductive development of peanut. Crop Sci 24:877882

Ketring DL, Brown RH, Sullivan GA, Johnson BB (1982) Growth physiology. In: Pattee HE, Young CT (eds) 
Peanut science and technology. Yoakum, Texas, pp 411457

Keuls M (1952) The use of the 'Studentized range' in connection with an analysis of variance. Euphytica 1:112-122

Koirala GP (1996) Collaborative research in Nepal: Needs and opportunities. In Gowda CLL, Ramakrishna A (eds) Summary proceedings of CLAN country coordinators' Steering committee meeting, ICRISAT, Patancheru, India, pp 39-43

Levene H (1960) Robust tests for equality of variances. In: Olkin I (ed) Contributions to probability and statistics: essays in honour of Harold Hotelling. Stanford University Press, Stanford, pp 278-292

Newman D (1939) The distribution of range in samples from a normal population expressed in terms of an independent estimate of standard deviation. Biometrika 31:20-30

Paterson HD, Williams ER (1976) A new class of resolvable incomplete block designs. Biometrika 63:395-400

Payne RW, Harding SA, Murray DA, Soutar DM, Baird DB, Welham SJ, Kane AF, Gilmour AR, Thompson R, Webster R, Wilson GT (2006) The guide to Genstat release 9, Part 2: statistics. VSN International, Hemel Hempstead
Sellschop JPF, Salmon SC (1928) The influence of chilling above the freezing point on certain crop plants. J Agric Res 37:315-338

Sinclair TR, Bennett JM, Boote KJ (1993) Leaf nitrogen content, photosynthesis and radiation use efficiency in peanut. Peanut Sci 20:40-43

Singh U, Jambunathan R (1980) Evaluation of rapid method for the estimation of protein content in chickpea (Cicer arietinum L.). J Sci Food Agric 31:247-254

Singleton JA, Pattee HE (1989) Effect of chilling injury on windrowed peanuts. Peanut Sci 16:51-54

Upadhyaya HD, Nigam SN, Singh S (2001) Evaluation of groundnut core collections to identify sources of tolerance to low temperature at germination. Indian J Plant Genet Resour 14:165-167

Upadhyaya HD, Ortiz R, Bramel PJ, Singh S (2003) Development of a core collection using taxonomical, geographical and morphological descriptors. Genetic Res Crop Evol 50:139-148

Ward J (1963) Hierarchical grouping to optimize an objective function. J Am Stat Assoc 38:236-244 